UDC 517.9

\title{
SUFFICIENT CONDITIONS FOR OSCILLATION OF SOLUTIONS OF FIRST ORDER NEUTRAL DELAY IMPULSIVE DIFFERENTIAL EQUATIONS WITH CONSTANT COEFFICIENTS
}

ДОСТАТНІ УМОВИ ДЛЯ ОСЦИЛЯЦЙНОСТІ РОЗВ'ЯЗКІВ
ДИФЕРЕНЦАЛЬНИХ РІВНЯНЬ ПЕРШОГО ПОРЯДКУ
ЗІ СТАЛИМИ КОЕФЦІЕТАМИ
ТА НЕЙТРАЛЬНИМ ЗАПЗНЕННЯМ ІМПУЛЬСНОЇ ДЇ

M. B. Dimitrova, V. I. Donev

Techn. Univ. Sliven

8800 Sliven, Bulgaria

e-mail: vanyodi@yahoo.com

This paper is dealing with the oscillatory properties of first order neutral delay impulsive differential equations and the corresponding inequalities with constant coefficients. The established sufficient conditions ensure oscillation of every solution of this type of the equations.

Розглянуто осциляційні властивості розв'язків диференціальних рівнянь першого порядку зі сталими коефіцієнтами та нейтральним запізненням імпульсної дї та відповідних до них нерівностей. Знайдені умови є достатніми для того, щзоб кожний розв'язок рівняння такого типу був осциляційним.

1. Introduction. In recent years, impulsive differential equations with deviating arguments (IDEDA) attract the attention of many mathematicians. Generally speaking, IDEDA are very interesting mixture of impulsive differential equations (see [1] and [2]) and differential equations with deviating argument (see [3-6]). The impulsive part of IDEDA reflects the discontinuities of first kind and can be used to model mathematically the short-time disturbances of some processes in the nature, whereas the differential part with its deviating argument (retarded, neutral, or advanced) is a very convenient instrument for a simulation of the dependance of the same processes on their history. Such processes occur in the theory of optimal control, theoretical physics, population dynamics, biotechnology, industrial robotics, etc. Among numerous papers published on IDEDA with retarded or advanced arguments, we choose to refer to [7-11].

Much less we know about the neutral impulsive differential equations (see [12-14]), i.e., the equations in which the highest-order derivative of the unknown function appears in the equation with the argument $t$ (the present state of the system), as well as with one or more retarded and/or advanced arguments (the past and/or the future state of the system).

In [14], the authors obtained some criteria for oscillation of the solutions of the neutral impulsive differential equation of first order $\left(E_{1}\right)$, where the coefficient $c$ in the neutral term belonged to the interval $(0,1)$. In the present paper we add and extend the results therein, allowing for the coefficient $c$ to be an arbitrary positive real number.

2. Preliminaries. Consider the first order neutral delay impulsive differential equation of the

(C) M. B. Dimitrova, V. I. Donev, 2010

ISSN 1562-3076. Нелінійні коливання, 2010, m. 13, Nㅇ 1 
form

$$
\begin{gathered}
\frac{d}{d t}[y(t)-c y(t-h)]+p y(t-\sigma)=0, \quad t \neq \tau_{k}, \quad k \in N, \\
\Delta\left[y\left(\tau_{k}\right)-c y\left(\tau_{k}-h\right)\right]+p_{k} y\left(\tau_{k}-\sigma\right)=0, \quad k \in N,
\end{gathered}
$$

as well as the corresponding inequalities,

$$
\begin{gathered}
\frac{d}{d t}[y(t)-c y(t-h)]+p y(t-\sigma) \leq 0, \quad t \neq \tau_{k}, \quad k \in N, \quad\left(N_{1, \leq}\right) \\
\Delta\left[y\left(\tau_{k}\right)-c y\left(\tau_{k}-h\right)\right]+p_{k} y\left(\tau_{k}-\sigma\right) \leq 0, \quad k \in N,
\end{gathered}
$$

and

$$
\begin{gathered}
\frac{d}{d t}[y(t)-c y(t-h)]+p y(t-\sigma) \geq 0, \quad t \neq \tau_{k}, \quad k \in N, \\
\Delta\left[y\left(\tau_{k}\right)-c y\left(\tau_{k}-h\right)\right]+p_{k} y\left(\tau_{k}-\sigma\right) \geq 0, \quad k \in N
\end{gathered}
$$

where the deviations $h$ and $\sigma$ and the coefficients $c, p$ and $p_{k}$ are positive constants. The points $\tau_{k} \in(0,+\infty), k \in N$, are fixed moments of the impulsive effect (let us call them jump points), where the unknown function reveals its first kind discontinuities as jumps. Here, in order to manifest the jumps of the unknown function $y(t)$ in such points of the impulsive effect, we use the notation

$$
\Delta\left[y\left(\tau_{k}\right)-c_{k} y\left(\tau_{k}-h\right)\right]=\Delta y\left(\tau_{k}\right)-c_{k} \Delta y\left(\tau_{k}-h\right), \quad \Delta y\left(\tau_{k}\right)=y\left(\tau_{k}+0\right)-y\left(\tau_{k}-0\right) .
$$

Denote by $P_{\tau} C(R, R)$ the set of all piecewise continuous on $\left(\tau_{k}, \tau_{k+1}\right], k \in N$, functions $u: R \rightarrow R$, which at the points $\tau_{k}$ are continuous from the left, i.e., $u\left(\tau_{k}-0\right)=\lim _{t \rightarrow \tau_{k}-0} u(t)=$ $=u\left(\tau_{k}\right)$, for which there exists a sequence of reals $\left\{u\left(\tau_{k}+0\right)\right\}$ such that $u\left(\tau_{k}+0\right)=\lim _{t \rightarrow \tau_{k}+0} u(t)$ and, which at the jump points $\tau_{k}, k \in N$, may have discontinuities of the first kind which we characterize as down-jumps if $\Delta u\left(\tau_{k}\right)=u\left(\tau_{k}+0\right)-u\left(\tau_{k}-0\right)<0$, or as up-jumps if $\Delta u\left(\tau_{k}\right)=u\left(\tau_{k}+0\right)-u\left(\tau_{k}-0\right)>0, k \in N$.

Introduce the following hypotheses:

$\left(\boldsymbol{H}_{1}\right) 0<\tau_{1}<\tau_{2}<\ldots<\tau_{k}<\ldots, \lim _{k \rightarrow+\infty} \tau_{k}=+\infty, \max \left\{\tau_{k+1}-\tau_{k}\right\}<+\infty, k \in N$;

$\left(\boldsymbol{H}_{2}\right) p>0, p_{k} \geq 0, k \in N, c>0$.

We will say that a function $y(t)$ is a solution of equation $\left(E_{1}\right)$, if there exists a number $T_{0} \in R$ such that $y \in P_{\tau} C\left(\left[T_{0},+\infty\right], R\right)$, the function $z(t)=y(t)-c y(t-h)$ is continuously differentiable for $t \geq T_{0}, t \neq \tau_{k}, k \in N$, and $y(t)$ satisfies equation $\left(E_{1}\right)$ for all $t \geq T_{0}$.

If not mentioned otherwise, we will assume throughout this paper that every solution $y(t)$ of equation $\left(E_{1}\right)$, that is under consideration here, is continuable to the right and is nontrivial. That is, $y(t)$ is defined on some ray of the form $\left[T_{y},+\infty\right)$ and $\sup \{|y(t)|: t \geq T\}>0$ for each $T \geq T_{y}$. Such a solution is called a regular solution of equation $\left(E_{1}\right)$.

We will say that a real-valued function $u$ defined on an interval of the form $[a,+\infty)$ has some property eventually, if there is a number $b \geq a$ such that $u$ has this property on the interval $[b,+\infty)$. 
A regular solution $y(t)$ of equation $\left(E_{1}\right)$ is said to be nonoscillatory, if there exists a number $t_{0} \geq 0$ such that $y(t)$ is of constant sign for every $t \geq t_{0}$. Otherwise, it is called oscillatory. Also, note that a nonoscillatory solution is called eventually positive (eventually negative), if the constant sign that determines its nonoscillation is positive (negative). Equation $\left(E_{1}\right)$ is called oscillatory, if all its solutions are oscillatory.

Moreover, writing a functional relation (or inequality), we will mean that it holds for all sufficiently large values of the argument.

In our investigations, we shall use two auxiliary functions composed from a solution of $\left(E_{1}\right)$. So, let us consider $y(t)$ as a solution of equation $\left(E_{1}\right)$ and set

$$
\begin{aligned}
& z(t)=y(t)-c y(t-h), \quad \Delta z\left(\tau_{k}\right)=\Delta y\left(\tau_{k}\right)-c \Delta y\left(\tau_{k}-h\right), \quad k \in N, \\
& w(t)=z(t)-c z(t-h), \quad \Delta w\left(\tau_{k}\right)=\Delta z\left(\tau_{k}\right)-c \Delta z\left(\tau_{k}-h\right), \quad k \in N .
\end{aligned}
$$

We introduce, at the beginning, two lemmas which investigate the asymptotic behavior of the functions $z(t)$ and $w(t)$ defined by $(*)$ and $(* *)$, if $y(t)$ is a non-oscillatory solution of $\left(E_{1}\right)$. The first one is formulated and proved for an eventually positive solution $y(t)$ of the equation $\left(E_{1}\right)$.

Lemma 1. Let $y(t)$ be an eventually positive solution of $\left(E_{1}\right)$ and the hypotheses $\left(H_{1}\right),\left(H_{2}\right)$ be satisfied. Then:

(a) $z(t)$ is an eventually decreasing function of $t$ with down-jumps;

(b) if $c \in(0,1]$, then $z(t)$ is an eventually positive function, i.e., $z(t)>0$ for all t large enough and $\lim _{t \rightarrow+\infty} z(t)=0$ with $\lim _{\tau_{k} \rightarrow+\infty}\left|\Delta z\left(\tau_{k}\right)\right|=0$;

(c) if $c>1$, then $z(t)$ is an eventually negative function, i.e., $z(t)<0$ for all $t$ large enough and $\lim _{t \rightarrow+\infty} z(t)=-\infty$.

Proof. (a) Let $y(t)$ be an eventually positive solution of the equation $\left(E_{1}\right)$, i.e., there exists $\tilde{t}>0$ such that $y(t)$ is defined for every $t \geq \tilde{t}$ and $y(t)>0, y(t-\sigma)>0, y(t-h)>0$ for $t \geq \tilde{t}+\max \{h, \sigma\}=t_{0}$. From $\left(E_{1}\right)$ and $(*)$ we have

$$
\begin{gathered}
z^{\prime}(t)=-p y(t-\sigma), \quad t \neq \tau_{k}, \quad k \in N, \quad t \geq t_{0}, \\
\Delta z\left(\tau_{k}\right)=-p_{k} y\left(\tau_{k}-\sigma\right), \quad k \in N, \quad \tau_{k} \geq t_{0} .
\end{gathered}
$$

It follows from (1) that $\Delta z\left(\tau_{k}\right)<0$, i.e., $z(t)$ has "down-jumps" at the points of impulsive effect $\tau_{k}$ and, because $z^{\prime}(t)<0$, we conclude that $z(t)$ is a decreasing function for $t \geq t_{0}$. The proof of (a) is complete.

(b) Let $y(t)$ be an eventually positive solution of $\left(E_{1}\right)$. Since (a) holds, then there exists $\lim _{t \rightarrow+\infty} z(t)$, which can be a positive number, zero, a negative number, or $-\infty$.

Assume that $z(t)<0$ eventually. Then, because $z(t)$ is a decreasing function with downjumps, for some $t_{1} \geq t_{0}$ there will exist $\delta_{\nu}>0$ such that $z(t)<-\delta_{\nu}$ for every $t \geq t_{1}, t \neq \tau_{k}$, $k \in N$, i.e.,

$$
y(t)-c y(t-h)<-\delta_{\nu}, \quad t \neq \tau_{k}, \quad t \geq t_{1} .
$$

Moreover, because the sequence of eventually negative numbers $\left\{z\left(\tau_{k}\right)\right\}_{k=1}^{+\infty}$ is decreasing, for our $\delta_{\nu}>0$, there will be such a term $\tau_{\nu}$ in the sequence of the impulsive moments $\left\{\tau_{k}\right\}$, whereafter $z\left(\tau_{k}\right)<-\delta_{\nu}$, for every $\tau_{k} \geq \tau_{\nu}$, when $k \geq \nu, k \in N, \nu \in N$. Hence,

$$
y\left(\tau_{k}\right)-c y\left(\tau_{k}-h\right)<-\delta_{\nu}, \quad \tau_{k} \geq \tau_{\nu}, \quad k \geq \nu, \quad k \in N, \quad \nu \in N .
$$


Denote $t_{\nu}=\max \left\{t_{1}, \tau_{\nu}\right\}$ and combine the last two inequalities as

$$
y(t)<-\delta_{\nu}+c y(t-h), \quad t \geq t_{\nu}
$$

which is obviously also fulfilled for $t=t_{\nu}$, too, i.e.,

$$
y\left(t_{\nu}\right)<-\delta_{\nu}+c y\left(t_{\nu}-h\right) .
$$

From (2), by iterations based on $t=t_{\nu}+(n-1) h, n \in N$, we get

$$
y(t)<-\delta_{\nu}\left(1+c+c^{2}+\ldots+c^{n-1}\right)+c^{n} y\left(t_{\nu}-h\right), \quad n \in N .
$$

When $c=1$, it follows from (3) that $y(t)$ becomes less than a negative number for fixed $y\left(t_{\nu}-h\right)$ and large enough $t$, which is a contradiction.

If $c \in(0,1)$, the inequality (3) implies, for large enough $t$, that

$$
y(t)<-\frac{\delta_{\nu}}{1-c}, \quad t \geq t_{\nu}
$$

and $y(t)$ becomes less than a negative number for large enough $t$, which is again a contradiction.

Hence, our assumption that eventually $z(t)<0$ is impossible and we conclude that eventually $z(t)>0$ and $\lim _{t \rightarrow+\infty} z(t)$ is a finite positive number or zero. The last fact implies

$$
\lim _{\tau_{k} \rightarrow+\infty}\left|\Delta z\left(\tau_{k}\right)\right|=0 .
$$

Further, assume $\lim _{t \rightarrow+\infty} z(t)=L, L>0$. Integrating $\left(E_{1}\right)$ from $t_{0}$ to $t$, we get

$$
\int_{t_{0}}^{t} z^{\prime}(r) d r+\int_{t_{0}}^{t} p y(r-\sigma) d r=0
$$

or

$$
z(t)-z\left(t_{0}\right)-\sum_{t_{0} \leq \tau_{k}<t} \Delta z\left(\tau_{k}\right)+\int_{t_{0}}^{t} p y(r-\sigma) d r=0,
$$

or

$$
z(t)=z\left(t_{0}\right)+\sum_{t_{0} \leq \tau_{k}<t} \Delta z\left(\tau_{k}\right)-\int_{t_{0}}^{t} p y(r-\sigma) d r .
$$

But $\Delta z\left(\tau_{k}\right)=-p_{k} y\left(\tau_{k}-\sigma\right)$ hence

$$
z(t)=z\left(t_{0}\right)-\sum_{t_{0} \leq \tau_{k}<t} p_{k} y\left(\tau_{k}-\sigma\right)-\int_{t_{0}}^{t} p y(r-\sigma) d r
$$


Because $z(t)=y(t)-c y(t-h)$, we have, in this case, that $L \leq z(t)<y(t)$, which determines $y(t)$ as a bounded function from below. Then (5) reduces to

$$
z(t) \leq z\left(t_{0}\right)-L\left[\sum_{t_{0} \leq \tau_{k}<t} p_{k}+\int_{t_{0}}^{t} p d r\right]
$$

which implies $\lim _{t \rightarrow+\infty} z(t)=-\infty$ and contradicts our assumption. Therefore, the only possibility left is $\lim _{t \rightarrow+\infty} z(t)=0$. The proof of (b) is complete.

(c) Proceeding as in the beginning of (b), we conclude that there exists $\lim _{t \rightarrow+\infty} z(t)$, which can be a positive number, zero, a negative number, or $-\infty$.

Assume $\lim _{t \rightarrow+\infty} z(t)=L>0$. Then we will have $L \leq z(t)<y(t)$. The last fact does determine $y(t)$ as a bounded function from below and if we integrate $\left(E_{1}\right)$ from $t_{0}$ to $t$, we can easily get (6), which will imply $\lim _{t \rightarrow+\infty} z(t)=-\infty$ and this will contradict our assumption.

Assume

$$
\lim _{t \rightarrow+\infty} z(t)=\lim _{t \rightarrow+\infty}[y(t)-c y(t-h)]=L=0 .
$$

It is obvious that then $z(t)>0$ eventually, i.e., there exists a number $t_{1} \geq t_{0}$, such that we have $y(t)>c y(t-h)>y(t-h)$ for every $t \geq t_{1}$. Observe that the last inequality holds as well as for those moments of impulsive effect $\tau_{k}$, for which $\tau_{k}>t_{1}, k \in N$. However, our assumption implies that there will exist a strictly increasing sequence $\left\{y\left(t_{n}\right)\right\}_{n=1}^{\infty}$ (with moments of impulsive effect $\tau_{k}$ eventually therein), which is bounded by a positive number, i.e., $\lim _{n \rightarrow+\infty} y\left(t_{n}\right)=K$, $K>0$ or which is unbounded, i.e., $\lim _{n \rightarrow+\infty} y\left(t_{n}\right)=+\infty$ and for which (7) has to be fulfilled. But for this sequence we have

$\lim _{t_{n} \rightarrow+\infty} z\left(t_{n}\right)=\lim _{t \rightarrow \infty}[y(t)-c y(t-h)]=\lim _{t_{n} \rightarrow+\infty} y\left(t_{n}\right)-c \lim _{t_{n} \rightarrow+\infty} y\left(t_{n}-h\right)=(1-c) \lim _{t_{n} \rightarrow+\infty} y\left(t_{n}\right)<0$

and the contradiction with (7) is evident, because $c>1$.

Finally assume $\lim _{t \rightarrow+\infty} z(t)=L<0$. Then, proceeding as in (b), we can get (2). Observe that the right-hand side of (2) has to be positive, because $y(t)$ is positive. So, we obtain the inequality $0<-\delta_{\nu}+c y(t-h)$, which clearly shows that $y(t)$ is a function bounded from below. Hence, if we integrate $\left(E_{1}\right)$ from $t_{0}$ to $t$, we can easily get (6), which will imply $\lim _{t \rightarrow+\infty} z(t)=-\infty$ contradicting our assumption again.

Thus, the above considerations imply that $\lim _{t \rightarrow+\infty} z(t)=-\infty$ and complete the proof of (c) and of the lemma.

The second lemma is only formulated for an eventually negative solution $y(t)$ of the equation $\left(E_{1}\right)$, but the proof is carried out analogously to the proof of Lemma 1.

Lemma 2. Let $y(t)$ be an eventually negative solution of $\left(E_{1}\right)$ and the hypotheses $\left(H_{1}\right),\left(H_{2}\right)$ be satisfied. Then:

(a) $z(t)$ is an eventually increasing function of t with up-jumps;

(b) if $c \in(0,1]$, then $z(t)$ is an eventually negative function, i.e., $z(t)<0$ for all large enough $t$ and $\lim _{t \rightarrow+\infty} z(t)=0$ with $\lim _{\tau_{k} \rightarrow+\infty}\left|\Delta z\left(\tau_{k}\right)\right|=0$; 
(c) if $c>1$, then $z(t)$ is an eventually positive function, i.e., $z(t)>0$ for large enough $t$ and $\lim _{t \rightarrow+\infty} z(t)=+\infty$.

Lemmas 1,2, applied to the functions $z(t)$ and $w(t)$ defined by $(*)$ and $(* *)$, where $y(t)$ is a non-oscillatory solution of $\left(E_{1}\right)$, lead to the following useful proposition.

Lemma 3. Let $y(t)$ be a solution of the equation $\left(E_{1}\right)$ and the hypotheses $\left(H_{1}\right),\left(H_{2}\right)$ be satisfied. Then:

(a) the functions $z(t)$ and $w(t)$ defined by $(*)$ and $(* *)$ are also solutions of $\left(E_{1}\right)$;

(b) for an eventually positive function $y(t), w^{\prime \prime}(t)$ is an eventually positive function;

(c) for an eventually negative function $y(t), w^{\prime \prime}(t)$ is an eventually negative function.

Proof. (a) A direct substitution of $z(t)$ defined by $(*)$ in the equation $\left(E_{1}\right)$ shows that $z(t)$ is a solution of $\left(E_{1}\right)$. The same holds for $w(t)$ defined by $(* *)$ as well. So, (a) is easily evident.

(b) According to (a), $z(t)$ defined by $(*)$ is a solution of equation $\left(E_{1}\right)$. So, we have

$$
[z(t)-c z(t-h)]^{\prime}=-[p z(t-\sigma)], \quad t \neq \tau_{k}
$$

From here, it is easy to see that

$$
w(t)^{\prime \prime}=[z(t)-c z(t-h)]^{\prime \prime}=-p[z(t-\sigma)]^{\prime}, \quad t \neq \tau_{k}
$$

From (8) and Lemma 1(a) one can easily derive (b).

(c) From (8) and Lemma 2(a) one can easily derive (c).

The lemma is proved.

In order to assist our investigations on the oscillation of $\left(E_{1}\right)$, we consider the delay impulsive differential equation with constant coefficients of the form

$$
\begin{gathered}
z^{\prime}(t)+Q z(t-s)=0, \quad t \neq \tau_{k}, \\
\Delta z\left(\tau_{k}\right)+q_{k} z\left(\tau_{k}-s\right)=0, \quad k \in N,
\end{gathered}
$$

and the corresponding to it inequalities

$$
\begin{gathered}
z^{\prime}(t)+Q z(t-s) \leq 0, \quad t \neq \tau_{k}, \quad\left(N_{2, \leq}\right) \\
\Delta z\left(\tau_{k}\right)+q_{k} z\left(\tau_{k}-s\right) \leq 0, \quad k \in N,
\end{gathered}
$$

and

$$
\begin{gathered}
z^{\prime}(t)+Q z(t-s) \geq 0, \quad t \neq \tau_{k}, \\
\Delta z\left(\tau_{k}\right)+q_{k} z\left(\tau_{k}-s\right) \geq 0, \quad k \in N,
\end{gathered}
$$

where the deviation $s$ and the coefficients $Q, q_{k}, k \in N$ are positive constants.

3. Oscillation of all solutions of the equation $\left(\boldsymbol{E}_{\mathbf{2}}\right)$. Let us note that in this section $z(t)$ is supposed to be self-contained and considered independently of what was set in (*). Our aim is to establish appropriate sufficient conditions under which the equation $\left(E_{2}\right)$ is oscillatory, in order to use this result in the next section. To this end, we introduce the following hypothesis: 
$\left(\boldsymbol{H}_{\mathbf{2}}^{*}\right) Q>0,1>q_{k} \geq 0, k \in N$.

The next lemma specifies a sufficient condition for equation $\left(E_{2}\right)$ to be oscillatory, which will be useful for investigation of oscillation of solutions of equation $\left(E_{1}\right)$.

Lemma 4. Assume that the hypotheses $\left(H_{1}\right)$ and $\left(H_{2}^{*}\right)$ are satisfied. Suppose also that

$$
\limsup _{t \rightarrow \infty}\left[\prod_{t-s \leq \tau_{k}<t}\left(1-q_{k}\right)\right]<e s Q, \quad k \in N .
$$

Then:

(a) the equation $\left(E_{2}\right)$ is oscillatory;

(b) the inequality $\left(N_{2, \leq}\right)$ has no eventually positive solutions;

(c) the inequality $\left(N_{2, \geq}\right)$ has no eventually negative solutions.

Proof. Since the proofs of (a), (b) and (c) can be carried out by similar arguments, it suffices to prove only the case (a). To this end, assume the converse that equation $\left(E_{2}\right)$ has a nonoscillatory solution. Since the negative of a solution of $\left(E_{2}\right)$ is again a solution of $\left(E_{2}\right)$, it suffices to prove the lemma considering this solution as an eventually positive function.

So, suppose that there exists a solution $z(t)$ of the equation $\left(E_{2}\right)$ and a number $\tilde{t}>0$ such that $z(t)$ is defined for $t \geq \tilde{t}$ and $z(t)>0, z(t-s)>0$ for $t \geq \tilde{t}+s=t_{0}$.

From $\left(E_{2}\right)$ and hypothesis $\left(H_{2}^{*}\right)$, it follows that

$$
\begin{gathered}
z^{\prime}(t)=-Q z(t-s)<0, \quad t \neq \tau_{k}, \quad k \in N, \\
\Delta z\left(\tau_{k}\right)=-q_{k} z\left(\tau_{k}-s\right)<0, \quad k \in N,
\end{gathered}
$$

i.e., $z(t)$ is a positive decreasing function for $t \geq t_{0}$ with "down-jumps"at the points of impulsive effect $\left(\Delta z\left(\tau_{k}\right)<0\right)$. At the beginning we can rearrange $\left(E_{2}\right)$, dividing by $z(t)$, in order to obtain

$$
\begin{gathered}
\frac{z^{\prime}(t)}{z(t)}=-Q \frac{z(t-s)}{z(t)}<-Q, \quad t \neq \tau_{k}, \quad k \in N, \\
\Delta z\left(\tau_{k}\right)=-q_{k} z\left(\tau_{k}-s\right)<-q_{k} z\left(\tau_{k}\right), \quad k \in N .
\end{gathered}
$$

It follows from Condition 1 , that there exists a constant $L>0$ and $t_{1} \geq t_{0}$ such that

$$
\frac{s Q}{m} \geq L>\frac{1}{e}, \quad t \geq t_{1}
$$

where we denote

$$
m=\limsup _{t \rightarrow \infty} \prod_{t \leq \tau_{k}<t+s}\left(1-q_{k}\right) .
$$

Now, integrate (9) from $t-s$ to $t$, i.e.,

$$
\int_{t-s}^{t} \frac{z(r)^{\prime}}{z(r)} d r<-\int_{t-s}^{t} Q d r
$$


and obtain

$$
\ln \frac{z(t)}{z(t-s)}+\sum_{t-s \leq \tau_{k}<t} \ln \frac{z\left(\tau_{k}\right)}{z\left(\tau_{k}+0\right)}<-s Q .
$$

Also, $z\left(\tau_{k}+0\right)-z\left(\tau_{k}\right)=-q_{k} z\left(\tau_{k}-s\right)<-q_{k} z\left(\tau_{k}\right)$ and $z\left(\tau_{k}+0\right)<\left(1-q_{k}\right) z\left(\tau_{k}\right)$, i.e., $\frac{1}{1-q_{k}}<\frac{z\left(\tau_{k}\right)}{z\left(\tau_{k}+0\right)}$. So, $\ln \frac{1}{1-q_{k}}<\ln \frac{z\left(\tau_{k}\right)}{z\left(\tau_{k}+0\right)}$ and from (10) and (11) we get

$$
\ln \left[\frac{z(t)}{z(t-s)} \prod_{t-s \leq \tau_{k}<t} \frac{1}{1-q_{k}}\right]<-s Q
$$

i.e.,

$$
\ln \left[\frac{z(t-s)}{z(t)} \prod_{t-s \leq \tau_{k}<t}\left(1-q_{k}\right)\right]>L m
$$

Using the inequality $e^{x}>e x$, it follows from the last inequality that

$$
\frac{z(t-s)}{z(t)} \prod_{t-s \leq \tau_{k}<t}\left(1-q_{k}\right)>e L m
$$

which implies

$$
\frac{z(t-s)}{z(t)}>e L
$$

Repeating the above procedure by induction (9), we conclude that there exists a sequence $\left\{t_{n}\right\}$, where $t_{n} \rightarrow \infty$ as $n \rightarrow \infty$, such that

$$
\frac{z(t-s)}{z(t)}>(e L)^{n}, \quad t \geq t_{n}
$$

Choose $n$ such that

$$
\left(\frac{2}{m L}\right)^{2}<(e L)^{n}
$$

which is possible because we have $e L>1$ by (10). Further, fix arbitrary chosen $\hat{t}$, where $\hat{t} \geq t_{n}$. Because of (10), there exists a $\xi \in[\hat{t}-s, \hat{t}]$ such that

$$
(\xi-\hat{t}+s) Q \geq \frac{m L}{2} \quad,(\hat{t}-\xi) Q \geq \frac{m L}{2} .
$$

Integrating $\left(E_{2}\right)$ over the interval $[\hat{t}-s, \xi]$, we find

$$
z(\xi)-z(\hat{t}-s)-\sum_{\hat{t}-s \leq \tau_{i} \leq \xi} \Delta z\left(\tau_{i}\right)+\int_{\hat{t}-s}^{\xi} Q z(r-s) d r=0
$$


or

$$
z(\xi)-z(\hat{t}-s)+\sum_{\hat{t}-s \leq \tau_{i} \leq \xi} q_{i} z\left(\tau_{i}-s\right)+Q \int_{\hat{t}-s}^{\xi} z(r-s) d r=0 .
$$

By omitting the first and the third terms and using the decreasing nature of $z(t)$ we find

$$
z(\hat{t}-s)>(\xi-\hat{t}+s) Q z(\xi-s)
$$

i.e.,

$$
\frac{z(\hat{t}-s)}{z(\xi-s)}>\frac{m L}{2}
$$

Similarly, integrating $\left(E_{2}\right)$ over the interval $[\xi, \hat{t}]$, we find

$$
z(\hat{t})-z(\xi)-\sum_{\xi \leq \tau_{i} \leq \hat{t}} \Delta z\left(\tau_{i}\right)+\int_{\xi}^{\hat{t}} Q z(r-s) d r=0
$$

or

$$
z(\hat{t})-z(\xi)+\sum_{\xi \leq \tau_{i} \leq \hat{t}} q_{i} z\left(\tau_{i}-s\right)+\int_{\xi}^{\hat{t}} Q z(r-s) d r=0 .
$$

By omitting the first and the third terms and using the decreasing nature of $z(t)$ we find

$$
z(\xi)>(\hat{t}-\xi) Q z(\hat{t}-s),
$$

i.e.,

$$
\frac{z(\xi)}{z(\hat{t}-s)}>\frac{m L}{2} .
$$

From (14) and (15) we conclude

$$
\frac{z(\xi-s)}{z(\xi)}<\left(\frac{2}{m L}\right)^{2}
$$

which, together with (12), imply

$$
(e L)^{n}<\frac{z(\xi-s)}{z(\xi)}<\left(\frac{2}{m L}\right)^{2} .
$$

Note that (16) is in contradiction with (13).

The lemma is proved.

4. Oscillation of all solutions of equation $\left(\boldsymbol{E}_{1}\right)$. Using the results of the previous two sections, we establish sufficient conditions for equation $\left(E_{1}\right)$ to be oscillatory. 
Theorem 1. Assume that the hypotheses $\left(H_{1}\right),\left(H_{2}\right)$ are satisfied. Suppose also that:

(a) $c<\frac{1}{p_{k}}, p_{k} \geq 1, k \in N$;

(b) $\limsup _{t \rightarrow \infty}\left[\prod_{t-h-\sigma \leq \tau_{k}<t}\left(1-c p_{k}\right)\right] \leq e c p(\sigma+h)$.

Then the equation $\left(E_{1}\right)$ is oscillatory.

Proof. Assume, conversely, that equation $\left(E_{1}\right)$ has a non-oscillatory solution. Since the negative of a solution of $\left(E_{1}\right)$ is again a solution of $\left(E_{1}\right)$, it suffices to prove the theorem considering an eventually positive solution of $\left(E_{1}\right)$.

So, let us suppose that there exists a solution $y(t)$ of the equation $\left(E_{1}\right)$ and a number $\tilde{t}>0$ such that $y(t)$ is defined for $t \geq \tilde{t}, y(t)>0$ for $t \geq \tilde{t}$ and $y(t-h)>0, y(t-\sigma)>0$ for $t \geq t_{0}=$ $=\tilde{t}+\max \{h, \sigma\}$. From Lemma 1(a), it follows that $z(t)$ which is defined by $(*)$ is a decreasing function for $t \geq t_{0}$ with "down-jumps" at the points of impulsive effect $\left(\Delta z\left(\tau_{k}\right)<0\right)$, and by Lemma 1(b), it follows that $z(t)$ is eventually positive.

Then, there exists some $t_{1} \geq t_{0}$ such that $z(t)>0$ for $t \geq t_{1} \geq t_{0}$ with "down-jumps"at the points of impulsive effect $\left(\Delta z\left(\tau_{k}\right)<0\right)$. From the fact that $y(t)-c y(t-h)=z(t)>0$, it is easy to conclude that $y(t)>z(t)$, as well as $y(t)>c y(t-h)$. Obviously, then we have $y(t-\sigma)>c y(t-\sigma-h)>c z(t-\sigma-h)$, i.e.,

$$
y(t-\sigma)>c z(t-\sigma-h)
$$

Multiplying both sides of (17) by $-p<0$ we obtain

$$
z^{\prime}(t)=-p y(t-\sigma)<-c p z(t-\sigma-h) .
$$

Hence,

$$
z^{\prime}(t)+c p z(t-\sigma-h)<0 .
$$

Observe that from (17) we have that $c z\left(\tau_{k}-\sigma-h\right)<y\left(\tau_{k}-\sigma\right), k \in N$.

Multiplying by $-p_{k}<0, k \in N$, both sides of the last inequality, we obtain that

$$
-c p_{k} z\left(\tau_{k}+h-\sigma\right)>-p_{k} y(\tau-\sigma)=\Delta z\left(\tau_{k}\right), \quad k \in N,
$$

i.e.,

$$
\Delta z\left(\tau_{k}\right)+c p_{k} z\left(\tau_{k}-\sigma-h\right)<0, \quad k \in N .
$$

From (18) and (19), denoting $s=\sigma+h>0, Q=c p, q_{k}=c p_{k}$, we see that the positive function $z(t)$ satisfies the delay impulsive differential inequality of the form

$$
\begin{gathered}
z(t)^{\prime}+Q z(t-s)<0, \quad t \neq \tau_{k}, \quad k \in N, \\
\Delta z\left(\tau_{k}\right)+q_{k} z\left(\tau_{k}-s\right)<0, \quad k \in N,
\end{gathered}
$$

which contradicts Lemma 4(b).

The theorem is proved.

As an immediate consequence of the above theorem, we formulate the following result. 
Corollary 1. Let the conditions of Theorem 1 be satisfied. Then:

(a) the inequality $\left(N_{1, \leq}\right)$ has no eventually positive solutions;

(b) the inequality $\left(N_{1, \geq}\right)$ has no eventually negative solutions.

The proof of the corollary is similar to that of Theorem 1 and is omitted.

Theorem 2. Assume that the hypotheses $\left(H_{1}\right),\left(H_{2}\right)$ are satisfied. Suppose also that:

(a) $\sigma>h>0$ and $c+p_{k}<1, k \in N$;

(b) $\limsup _{t \rightarrow \infty}\left[\prod_{t+h-\sigma \leq \tau_{k}<t}\left(1-\frac{p_{k}}{1-c}\right)\right] \leq \frac{e p(\sigma-h)}{1-c}$.

Then the equation $\left(E_{1}\right)$ is oscillatory.

Proof. Assume, for the sake of contradiction, that equation $\left(E_{1}\right)$ has an eventually positive solution $y(t)$. Then there exists $\tilde{t}>0$ such that $y(t)>0$ for every $t>\tilde{t}$. Also, there is $t_{0} \geq \tilde{t}+\sigma$ such that $y(t-\sigma)>0$ and $\Delta\left[y\left(\tau_{k}\right)-c y\left(\tau_{k}-h\right)\right]=-p_{k} y\left(\tau_{k}-\sigma\right)<0, k \in N$, for every $t \geq t_{0}$. Now, by the conditions of the theorem, Lemma 3(a) and Lemma 1(b), it follows that the functions $z(t)$ and $w(t)$, defined respectively by $(*)$ and $(* *)$, will be eventually positive decreasing solutions to the equation $\left(E_{1}\right)$ for every $t \geq t_{0}$. That is, $w(t)$ satisfies, as a decreasing positive solution, the equation

$$
\begin{gathered}
\frac{d}{d t}[w(t)-c w(t-h)]+p w(t-\sigma)=0, \quad t \neq \tau_{k}, \\
\Delta\left[w\left(\tau_{k}\right)-c w\left(\tau_{k}-h\right)\right]+p_{k} w\left(\tau_{k}-\sigma\right)=0, \quad k \in N .
\end{gathered}
$$

Let us recall that, by Lemma 3(b), $w^{\prime}(t)$ is an increasing function. Therefore, from (21) it is easy to see that

$$
\begin{aligned}
w^{\prime}(t-h) & -c w^{\prime}(t-h)+p w(t-\sigma) \leq \\
& \leq w^{\prime}(t)-c w^{\prime}(t-h)+p w(t-\sigma)= \\
& =\frac{d}{d t}[w(t)-c w(t-h)]+p w(t-\sigma)=0 .
\end{aligned}
$$

Moreover, since $z(t)$ is a decreasing function, we see that $z\left(\tau_{k}-\sigma\right)<z\left(\tau_{k}-\sigma-h\right)$ and so, using the definitions of the functions $z(t)$ and $w(t)$, it is easy to conclude that

$$
\Delta w\left(\tau_{k}\right)=-p_{k} z\left(\tau_{k}-\sigma\right)>-p_{k} z\left(\tau_{k}-\sigma-h\right)=\Delta w\left(\tau_{k}-h\right), \quad k \in N .
$$

So, in view of the above observation, from (21) it follows that, for each $k \in N$,

$$
\begin{aligned}
\Delta w\left(\tau_{k}-h\right) & -c \Delta w\left(\tau_{k}-h\right)+p_{k} w\left(\tau_{k}-\sigma\right) \leq \\
& \leq \Delta w\left(\tau_{k}\right)-c \Delta w\left(\tau_{k}-h\right)+p_{k} w\left(\tau_{k}-\sigma\right)= \\
& =\Delta\left[w\left(\tau_{k}\right)-c w\left(\tau_{k}-h\right)\right]+p_{k} w\left(\tau_{k}-\sigma\right)=0 .
\end{aligned}
$$

Now, from (22) and (23), it follows that $w(t)$ is an eventually positive function for which

$$
(1-c) w^{\prime}(t-h)+p w(t-\sigma) \leq 0, \quad t \neq \tau_{k},
$$




$$
(1-c) \Delta w\left(\tau_{k}-h\right)+p_{k} w\left(\tau_{k}-\sigma\right) \leq 0, \quad k \in N
$$

Divide the last two inequalities by $1-c>0$ and denote $s=\sigma-h, Q=\frac{p}{1-c}, q_{k}=\frac{p_{k}}{1-c}$. Then, substituting $\hat{t}=t-h$ we conclude that $w(t)$ becomes an eventually positive solution of the delay impulsive differential inequality of the form

$$
\begin{gathered}
w^{\prime}(\hat{t})+Q w(\hat{t}-s) \leq 0, \quad t \neq \tau_{k}, \\
\Delta w\left(\tau_{k}\right)+q_{k} w\left(\tau_{k}-s\right) \leq 0, \quad k \in N .
\end{gathered}
$$

But the last conclusion, contradicts Lemma 4(b) and completes the proof.

As an immediate consequence of the above theorem, we formulate the following result.

Corollary 2. Let the conditions of Theorem 2 be satisfied. Then:

(a) the inequality $\left(N_{1, \leq}\right)$ has no eventually positive solutions;

(b) the inequality $\left(N_{1, \geq}\right)$ has no eventually negative solutions.

The proof of the corollary is similar to that of Theorem 2 and is omitted.

Note that the previous two theorems considered the oscillation of the equation $\left(E_{1}\right)$ under the condition $c \in(0,1)$. Next we give a result in the case where $c>1$.

Theorem 3. Assume that the hypotheses $\left(H_{1}\right)-\left(H_{2}^{*}\right)$ are satisfied. Suppose also that:

(a) $\sigma \leq h, c>1$;

(b) $\liminf _{t \rightarrow+\infty}\left[p(h-\sigma)+\sum_{t+\sigma \leq \tau_{k} \leq t+h} p_{k}\right] \geq c^{2}$.

\section{Then equation $\left(E_{1}\right)$ is oscillatory.}

Proof. Assume, for the sake of contradiction, that $y(t)$ is an eventually positive solution of the equation $\left(E_{1}\right)$. Then, in view of Lemma 3(a) and Lemma 1(c), the function $z(t)$, which is defined by $(*)$, will be an eventually negative decreasing solution to the equation $\left(E_{1}\right)$. That is,

$$
\begin{gathered}
{[z(t)-c z(t-h)]^{\prime}+p z(t-\sigma)=0, \quad t \neq \tau_{k},} \\
\Delta\left[z\left(\tau_{k}\right)-c z\left(\tau_{k}-h\right)\right]+p_{k} z\left(\tau_{k}-\sigma\right)=0, \quad k \in N .
\end{gathered}
$$

Integrating (25) from $t+\sigma$ to $t+h$, we obtain

$$
z(t+h)-z(t+\sigma)-c[z(t)-z(t+\sigma-h)]+\sum_{t+\sigma \leq \tau_{k} \leq t+h} p_{k} z\left(\tau_{k}-\sigma\right)+\int_{t+\sigma}^{t+h} p z(r-\sigma) d r=0 .
$$

By omitting the first term and counting the decreasing nature of $z(t)$ we have

$$
-z(t+\sigma)-c z(t)+c z(t+\sigma-h)+\sum_{t+\sigma \leq \tau_{k} \leq t+h} p_{k} z\left(\tau_{k}-\sigma\right)+p(h-\sigma) z(t)>0,
$$

or, because $z(t)<z(t-h)$, we can get

$$
-z(t+\sigma)-c z(t)+c z(t+\sigma-h)+\sum_{t+\sigma \leq \tau_{k} \leq t+h} p_{k} z\left(\tau_{k}-\sigma\right)+p(h-\sigma) z(t-h)>0 .
$$


Observe that $w(t)$ defined by $(* *)$ will be a positive function of $t$, by Lemma 2(c). Therefore, $w(t)=z(t)-c z(t-h)>0$ leads us to the conclusion that $z(t)>c z(t-h)$. Hence,

$$
-c z(t)<-c^{2} z(t-h) \text { and }-z(t+\sigma)<-c z(t+\sigma-h) .
$$

From (26) and (27), it follows that

$$
-c z(t+\sigma-h)-c^{2} z(t-h)+c z(t+\sigma-h)+\sum_{t+\sigma \leq \tau_{k} \leq t+h} p_{k} z\left(\tau_{k}-\sigma\right)+p(h-\sigma) z(t-h)>0 .
$$

Moreover, because of the decreasing nature of $z(t)$, we have $z\left(\tau_{k}-\sigma\right) \leq z(t)<z(t-h)$ for $t+\sigma \leq \tau_{k} \leq t+h$ and so, from (28), we derive

$$
-c^{2} z(t-h)+z(t-h) \sum_{t+\sigma \leq \tau_{k} \leq t+h} p_{k}+p(h-\sigma) z(t-h)>0,
$$

or, finally,

$$
z(t-h)\left[-c^{2}+p(h-\sigma)+\sum_{t+\sigma \leq \tau_{k} \leq t+h} p_{k}\right]>0,
$$

which, because of the negative function $z(t)$, implies

$$
p(h-\sigma)+\sum_{t+\sigma \leq \tau_{k} \leq t+h} p_{k}<c^{2} .
$$

The conclusion obtained contradicts Condition 2 of the theorem and completes the proof.

As an immediate consequences of the above theorem, we get the following results.

Theorem 4. Assume that the hypotheses $\left(H_{1}\right)-\left(H_{2}^{*}\right)$ are satisfied. Suppose also that:

(a) $\sigma \leq h, c>1$

(b) $0 \leq p(h-\sigma) \leq \frac{c}{e}$;

(c) $\liminf _{t \rightarrow+\infty} \sum_{t+\sigma \leq \tau_{k} \leq t+h} p_{k} \geq c^{2}$.

Then equation $\left(E_{1}\right)$ is oscillatory.

Remark 1. As is known (see, for example, [3], Corollary 3.1.6, or [5], Theorem 6.4.2), a sufficient condition, for oscillation of the neutral delay differential equation

$$
\frac{d}{d t}[y(t)-c y(t-h)]+p y(t-\sigma)=0
$$

without impulse effects, is $\frac{p}{c}(h-\sigma)>\frac{1}{e}$. However, the presence of the impulse effects in the differential equation can cause or destroy the oscillation of its solutions. Our result above demonstrates in partiular the influence of the appearance of the impulse effects on the behavior of solutions of $\left(E_{1}\right)$. Indeed, Theorem 4 shows that the neutral delay impulsive differential equation $\left(E_{1}\right)$ is oscillatory even in the case where $\frac{p}{c}(h-\sigma) \leq \frac{1}{e}$. 
Corollary 3. Let the conditions of Theorem 3 or Theorem 4 be satisfied. Then:

(a) the inequality $\left(N_{1, \leq}\right)$ has no eventually positive solutions;

(b) the inequality $\left(N_{1, \geq}\right)$ has no eventually negative solutions.

5. Examples. Example 1. Consider the neutral delay impulsive differential equation

$$
\begin{gathered}
{[y(t)-0.2 y(t-2)]^{\prime}+\frac{1}{10} y(t-1)=0, \quad t \neq \tau_{k},} \\
\Delta\left[y\left(\tau_{k}\right)-0.2 y\left(\tau_{k}-2\right)\right]+y\left(\tau_{k}-1\right)=0, \quad k \in N,
\end{gathered}
$$

where $p=\frac{1}{10}, p_{k}=1, c=0.2, h=2>\sigma=1$ and $\tau_{k+1}-\tau_{k}=1, k \in N, e=\exp (1)$.

Here, the hypotheses $\left(H_{1}\right),\left(H_{2}\right)$ are satisfied and $c \in(0,1)$. It is easy to check that the conditions of Theorem 1 are satisfied. Indeed, $c=0.2<\frac{1}{p_{k}}=1, p_{k} \geq 1, k \in N$, and $\limsup _{t \rightarrow \infty}\left[\prod_{t-h-\sigma \leq \tau_{k}<t}\left(1-c p_{k}\right)\right]=\limsup _{t \rightarrow \infty}\left[\prod_{t-3 \leq \tau_{k}<t}(0.8)\right]=0.64 \leq e c p(\sigma+h)=0.6 e$.

Therefore, by Theorem 1, all solutions of the above equation are oscillatory. For example, one oscillatory solution of this equation is the "pulsatile exponent"(see, [12] for the definition of such a "pulsatile"function) $y(t)=A^{i\left[t_{0}, t\right)} e^{-\lambda_{*} t}, t_{0} \geq 0$, where $A=-0.2226870709$ is the "pulsatile constant" and $\lambda_{*}=0.12226870709$ is the exponential argument.

Example 2. Consider the neutral delay impulsive differential equation

$$
\begin{gathered}
{[y(t)-1.5 y(t-2)]^{\prime}+\frac{1}{e} y(t-1)=0, \quad t \neq \tau_{k},} \\
\Delta\left[y\left(\tau_{k}\right)-1.5 y\left(\tau_{k}-2\right)\right]+4 y\left(\tau_{k}-1\right)=0, \quad k \in N,
\end{gathered}
$$

where $p=\frac{1}{e}, p_{k}=4, c=1.5, h=2>\sigma=1$ and $\tau_{k+1}-\tau_{k}=1, k \in N, e=\exp (1)$.

Here, the hypotheses $\left(H_{1}\right),\left(H_{2}\right)$ are satisfied and $c \in(1,+\infty)$. It is easy to check that the conditions of Theorem 3, as well as the conditions of Theorem 4 are satisfied. Indeed,

$\liminf _{t \rightarrow+\infty}\left[p(h-\sigma)+\sum_{t+\sigma \leq \tau_{k} \leq t+h} p_{k}\right]=\liminf _{t \rightarrow+\infty}\left[\frac{1}{e}(2-1)+\sum_{t+1 \leq \tau_{k} \leq t+2} 4\right]=4+\frac{1}{e} \geq c^{2}=2.25$.

Therefore, by Theorem 3, or by Theorem 4, all solutions of the above equation are oscillatory. For example, one oscillatory solution of this equation is the "pulsatile exponent" $y(t)=$ $=A^{i\left[t_{0}, t\right)} e^{-\lambda_{*} t}, t_{0} \geq 0$, where $A=-0.5698188218$ is the "pulsatile constant"and $\lambda_{*}=$ $=0.14437601777$ is the exponential argument.

6. Acknowledgement. The authors dedicate this work to the memory of their teacher Dr. M. K. Grammatikopulos.

1. Lakshmikantham V., Bainov D. D. , Simeonov P. S. Theory of impulsive differential equations. - Singapore: World Sci., 1989. 
2. Samoilenko A. M., Perestyuk N. A. Differential equations with impulse effect (in Russian). - Kiev: Vyshcha Shkola, 1987.

3. Erbe L.H., Kong Q., Zhang B.G. Oscillation theory for functional differential equations. - New York: Marcel Decker Inc., 1995.

4. Grammatikopulos M. K., Grove E. A., Ladas G. Oscillation and asymptotic behavior of neutral differential equations with deviating arguments // Appl. Anal. - 1986. - 22. - P. 1 -19.

5. Gyori I., Ladas G. Oscillation theory of delay differential equations with applications. - Oxford: Clarendon Press, 1991.

6. Ladde G.S., Lakshmikantham V., Zhang B. G. Oscillation theory of differential equations with deviating arguments // Pure and Appl. Math. - 1987. - 110.

7. Boichuk A., Covachev V. Periodic solutions of impulsive systems with a small delay in the critical case of second order // Nonlinear Oscillations. - 1998. - 1, № 1. - P. 6-19.

8. Grammatikopulos M. K., Dimitrova M. B., Donev V.I. Oscillations of first order delay impulsive differential equations // Techn. Rept. Univ. Ioannina, Greece. - 2007. - 16. - P. 171-182.

9. Grammatikopulos M. K., Dimitrova M. B., Donev V.I. Oscillations of first order impulsive differential equations with variable coefficients and advanced argument // Proc. 33-rd Conf. (AMEE'07)/ Ed. M. D. Todorov. - 2007. - P. 206-214.

10. Gopalsamy K., Zhang B. G. On delay differential equations with impulses // J. Math. Anal. and Appl. 1989. - 139. - P. $110-122$.

11. Zhang Y., Zhao A., Yan J. Oscillation criteria for impulsive delay differential equations // Ibid. - 1997. 205. - P. $461-470$.

12. Dimitrova M. B., Donev V. I. Existence and asymptotic behavior of the positive solutions of neutral impulsive differential equations // Nonlinear Oscillations. - 2005. - 8, № 3. - P. 304-318.

13. Graef J. R., Shen J. H., Stavroulakis I. P. Oscillation of impulsive neutral delay differential equations // J. Math. Anal. and Appl. - 2002. - 268, № 1. - P. 310-333.

14. Grammatikopulos M. K., Dimitrova M. B., Donev V.I. Oscillations of first order neutral impulsive delay differential equations with constant coefficients // Techn. Rept. Univ. Ioannina, Greece. - 2007. - 16. - P. 183 193.

Received 05.11.08 